\title{
An Optimal Path Scheduling Approach in Wireless Sensor Network
}

\author{
Ashish Sharma \\ Student, M.Tech (IT) \\ Department of CSE/IT \\ ITM, University, \\ Gurgaon, India
}

\author{
Shilpa Mahajan \\ Asstt. Professor \\ Department of CSE/IT \\ ITM, University, \\ Gurgaon, India
}

\begin{abstract}
One of the major challenges for any network is the effective routing to perform reliable communication over the network. This challenge becomes more critical for sensor network where each node is having the limited energy. To provide the reliable communication a distance and energy based optimal path is selected by most of the authors. But if the communication is performed regularly over such path, the energy of all intermediate nodes will be degraded and it will disturb the energy balance over the network. To provide balanced energy consumption, a time sliced based alternate path selection approach is suggested in this work. In this paper, a novel path switching approach is suggested to provide the energy balanced routing over the network.
\end{abstract}

\section{Keywords}

Energy Balanced, Alternate Path, Effective Routing.

\section{INTRODUCTION}

A sensor network is one of the most growing network phenomenons that are having its importance in different real time applications. Sensor network has become the part of many application areas like vehicular network, medical application, body area network etc. With the increase of its importance, lot of advancement is already done in the sensor network in terms of its capacity, low power functionality, multifunctional nodes, low cost, smaller size etc. These sensor nodes are capable to communicate effectively for small size network and for short distances. One of such advancement is represented by the smart sensor nodes called smart dust. Smart dust has become most effective sensor devices that provide the autonomous sensing and computing along with memory storage.

Smart dust provides the easy way communication within the network as well as provides the interaction with other connected nodes or devices. The main advantage of the sensor nodes is its continuous monitoring capability, data gathering and the intelligent decision making capability. The basic properties of smart sensors is shown in figure 1.

Smart Dust networks are more effective than the traditional network as it provides the low power communication over the network with effective utilization of memory and capable to take dynamic decisions. These constraints show that the network is capable to provide the effective data propagation and the routing. The main features of the sensor network, that make it significantly effective low power communication, are given as under.

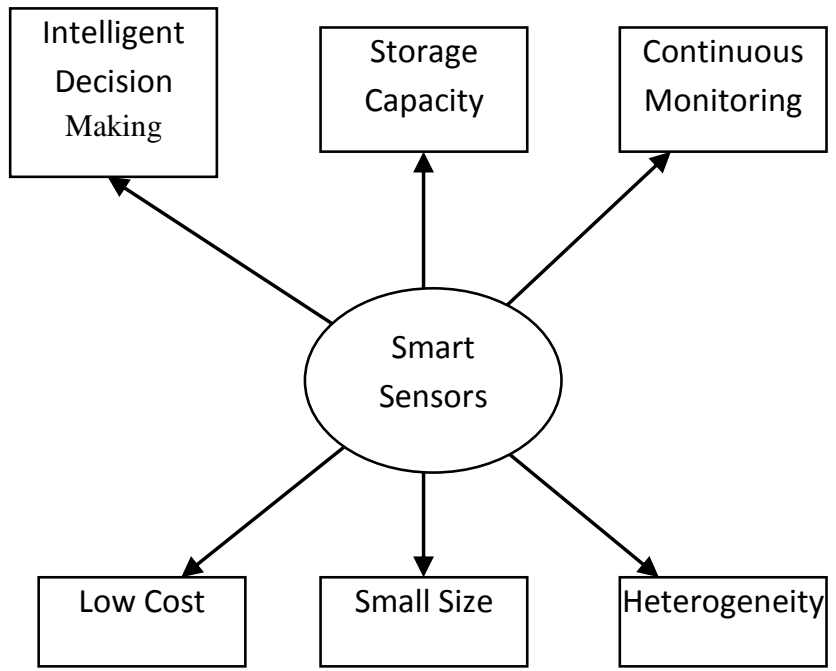

Fig 1 : Capabilities of Smart Sensor

(i) Size: As the sensor network is provides intelligent decision making and memory storage, it able to handle large network with thousands of nodes. It can also be established in a network with large physical area as the power consumption in multi-hop communication is also lesser than traditional networks.

(ii) Mobility: In most of the cases, the sensor nodes are placed at fixed location. But smart sensors can be used in applications where the node mobility is required such as underwater sensor network, vehicular network etc.

(iii) Information Transfer: Smart Sensors can be deployed in such harsh environment and unexpected situations where the node failure is common such as sensor node placement in battlefield. In such networks, sensor node provides the information saving and transfer, if the node life is expectedly lesser.

(iv) Improved Life Span: Even the sensor nodes are defined with limited energy specification and with each communication some energy loss occur. Smart sensors are capable to improve the node life because of its storage capability, lesser power consumption capability.

(v) Dynamic Decision: Smart sensors are capable to take the dynamic decisions about the information transfer.

The major challenge for the smart sensor network is to provide the reliable communication along with lesser power consumption. The basic characteristics of reliable communications given as under : 


\subsection{Reliable Routing}

End-to-End Communication: The foremost requirement of smart sensor network is to provide high throughput. The end to end packet delivery must be higher and the packet loss over the network should be minimum.

Lesser Conflict: If the network is performing the multi path communication, the confliction should be lesser. It means the path selection must be effective so that the node repetition as the intermediate nodes will be minimum.

Lesser Latency: A sensor network can have congested communication but in all cases, the latency over the communication should be lesser. If the communication is performed over the alternate path, even then the communication delay should be lesser.

In this paper, an improved routing scheme is suggested under the capabilities of the sensor network. In this section, a brief introduction to sensor network and smart sensor network is defined. The work also defined the properties of reliable routing and reliable network. In section II, the work already done in the area of shortest path routing and alternate path routing is defined. In section III, the presented work methodology and routing scheme is explored. In section IV, the conclusion driven from the work is defined.

\section{LITERATURE SURVEY}

Lot of work is already done to optimize the routing in mobile and sensor network. Some of work done by the earlier authors to optimize the routing is described in this section. In year 2003, Chao Gui has defined an optimized routing approach for the self healed mobile network. Author defined the optimality under different communication metrics such as energy consumption, route length, number of intermediate nodes, energy awareness and the load analysis. Author defined a framework to provide the optimize route selection in mobile network. Author defined a subpath analysis based scheme under the node monitoring scheme to optimize the routing[1]. Another work on region based routing was defined by Hao Wen in year 2009. Author defined a region aware storage friendly approach for route generation. Author defined a history analysis approach so that the node tracking will be done effectively and a parametric analysis will be performed under the mobility model to provide effective routing in vehicular network[2]. A work on on-demand routing approach was proposed by Jiejun Kong in year 2003. Author defined the effective routing in hostile critical environment. Author focused on two main network problems called route anonymity and the back flow effecting destination effective routing so that the location privacy will be achieved. Author defined the routing in real scenarios[3].

To provide the secure routing in indoor environment, KyuHwan Lee, presented an authentication specification routing scheme. Author defined the work to achieve the high performance and the secure transmission. The analytical work is also defined by the author to achieve the reliable communication over the network[4]. Another work to improve the routing performance in mobility sensitive mobile network by Athanasios Bamis in year 2006.Author defined the communication under the standard and effective properties of mobile network as well as provides the analysis at different speed mobile nodes. Author defined the key authentication to elect the reliable node for communication[5]. In year 2009, Khaleel Ur Rahman defined an integrated routing approach for mobile network. Author defined the bidirectional analysis in infrastructure based mobile network. Author defined a bridge oriented gateway selection to provide effective routing over the network[6]. S.Satish provides the route optimization using Ant Colony approach. Author defined the cache oriented analysis under the source initiated routing approach so that adaptive energy optimization will be achieved[7]

In year 2011, Giovanni Comarela has defined the robot routing in sensor network under the Ant Colony optimization. Author defined the inclusion of travelling salesman approach to provide the effective routing and also provide the improvement by using the heuristic search. Author defined the comparative analysis with different approaches [8]. Another work in same year was proposed by C. D'Souza to obtain the energy effectiveness by performing the effective node placement in sensor network. Author used the swarm optimize approach to reduce the energy consumption[9]. A route selection based routing protocol was suggested by Taesoo Jun in year 2007. Author presented the network deployment under the dependentcy analysis so that the effective supporting routing will be obtained under the performance analysis, cost analysis and the lesser delay[10]. In year 2012, Jing-Hui Zhong has defined ACO based routing approach to improve the network life and to increase the network communication. Author utilizes the network characteristics in an effective way and increase the network responsiveness[11]. To provide the attack preventive routing Mohsen Saffarian has defined an ACO based approach in year 2008. Author defined the work to migigate the network threat and improves the network communication. Author defined the agent based approach to provide secure and reliable routing[12].

\section{RESEARCH METHODOLOGY}

In this paper, an effective reliable, efficient and energy effective routing approach is suggested in this work. The presented work is defined on a smart sensor network in which each node is defined with some smart capabilities. The most effective properties of these sensors are the storage capability and the ability to take the decision making along with integrated processing. The main base of the presented work is to provide the energy balanced communication over the network. To provide this balancing, instead of communicating the data over a fixed static path, an array of the network paths is defined in this work. As the nodes are defined with memory specification, at the earlier stage, all the possible paths are generated and stored in the available memory. These paths include the shortest path as well as alternate paths under the energy effectiveness and the load effective analysis. These paths are generated in such a way that the intermediate nodes of a path will not be included in other path. Now these paths get activated in a sequence so that each time a separate path will be elected for the communication. A time-slicing approach is suggested in this work to activate these stored paths. The main objective of the work is improve the network life and to provide the reliable communication over the network. In this section, the assumption, working and the algorithmic approach of the presented work is explained in detail

\subsection{Assumptions}

a) All the network nodes are defined at static location.

b) The base station is placed at one end of the network

c) Single source and destination points are described so that effective route will be generated. 
d) Nodes are defined with energy specification and with each participation some energy loss will be done.

e) All the sensor nodes are homogenous but they are having different congestion factor and the energy level.

f) All nodes are having some memory to store the associated paths.

g) Nodes are defined with decision capability about the next hop selection.

h) Each node is defined with a bit as the route participation node.

\subsection{Simulation Environment}

The presented work will be implemented in matlab environment. To design the sensor network, it is required to setup the energy parameters for sensor nodes present in the network. The standard parameters that will be considered in the work are listed here under.

Table 2 : Network Parameters

\begin{tabular}{|c|c|}
\hline Parameters & Values \\
\hline Area & $100 \times 100$ \\
\hline Topology & Random \\
\hline Base Station Position & Random \\
\hline Energy & $1 \mathrm{~J}$ \\
\hline Transmission Energy & $5 \mathrm{~nJ}$ \\
\hline Receiving Energy & $5 \mathrm{~nJ}$ \\
\hline Forwarding Energy & $1 \mathrm{~nJ}$ \\
\hline Aggregative Energy & $.01 \mathrm{~nJ}$ \\
\hline
\end{tabular}

To begin the work, the network is been established under these parameters. The network diagram of the presented work is shown in fig 2.

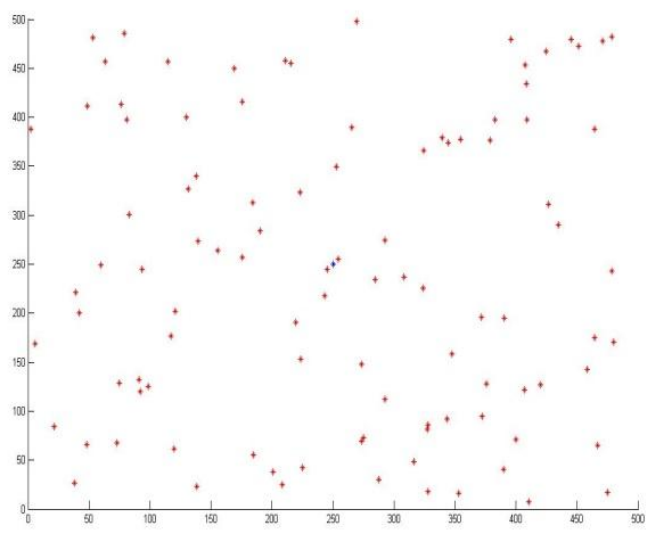

Fig 2 : Network Design

As shown in the figure, red dots are showing the sensor nodes distributed over the network and blue node is representing the base station.

\subsection{Work Description}

The main idea of the work is to provide the switching path capability so that the load over a particular path node will not be increased. The work includes the route generation under different parameters at the initial phase and stores them in the memory of source node. Now activate these available paths one by one under the time slicing mechanism so that the no load on any intermediate node will be increased and an effective communication will be done over the network. The main stages of the presented work are shown in figure 2 .

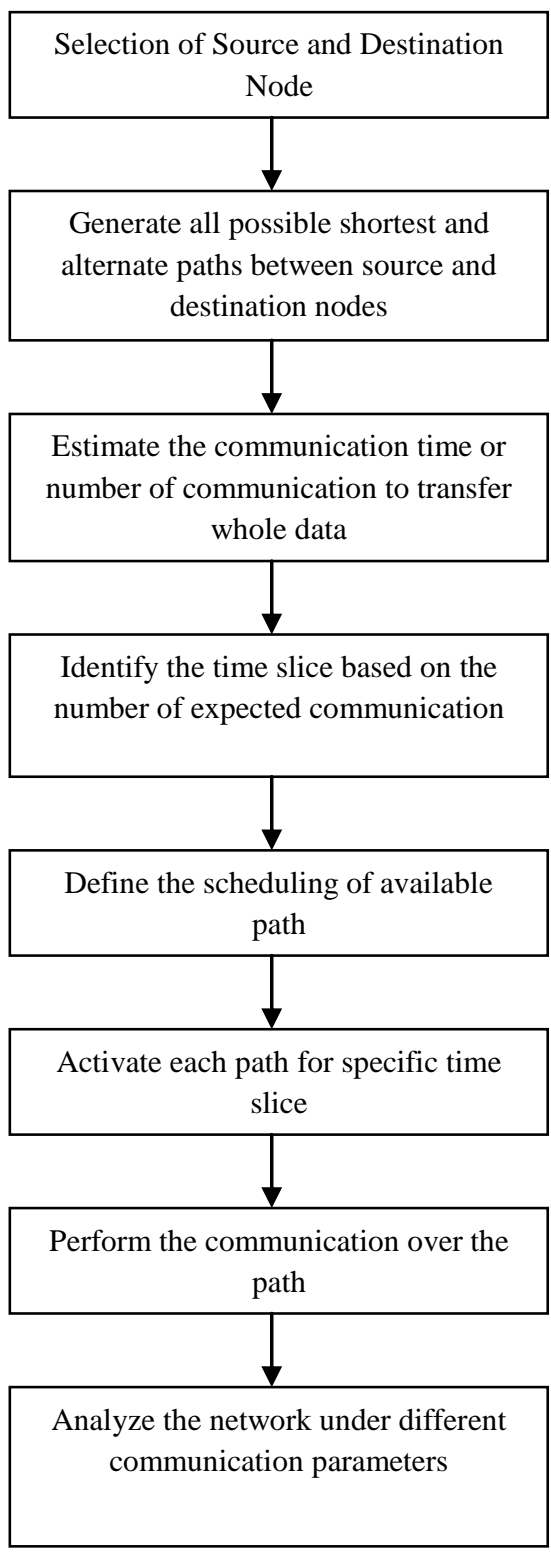

Fig 2. Process Flow 


\section{CONCLUSION}

A wireless network suffers from the problem of congestion when many nodes selects the same favorite shortest path as the communication route. In sensor network, this congestion problem is more critical. In this presented work, an effective alternate path based energy balanced routing is suggested. This work is based on the smart sensor network where each node is capable to store the possible paths and these nodes get activated under the scheduling machismo. The work is expected to improve the network life by providing the effective routing.

\section{REFERENCES}

[1] Chao Gui," SHORT: Self-Healing and Optimizing Routing Techniques for Mobile Ad Hoc Networks", MobiHoc'03, June 1-3, 2003, Annapolis, Maryland, USA. ACM 1-58113-684-6/03/0006

[2] Hao Wen," RENA: Region-based Routing in Intermittently Connected Mobile Network", MSWiM'09, October 26-29, 2009, Tenerife, Canary Islands, Spain. ACM 978-1-60558-616-9/09/10

[3] Jiejun Kong," ANODR: ANonymous On Demand Routing with Untraceable Routes for Mobile Adhoc Networks", MobiHoc'03, June 1-3, 2003, Annapolis, Maryland, USA ACM 1-58113-684-6/03/0006

[4] Kyu-Hwan Lee," Routing based Authentication for Mobile Ad hoc Network in Home Environment".

[5] Athanasios Bamis," A Mobility Sensitive Approach for Efficient Routing in Ad Hoc Mobile Networks", MSWiM'06, October 2-6, 2006, Torremolinos, Malaga, Spain. ACM 1-59593-477-4/06/0010
[6] Khaleel Ur Rahman Khan," An Efficient Integrated Routing Protocol for Interconnecting Mobile Ad Hoc Networks and the Internet", International Conference on Advances in Computing, Communication and Control (ICAC3'09) ICAC3'09, January 23-24, 2009, Mumbai, Maharashtra, India. ACM 978-1-60558-351-8

[7] S. Sathish," Cache Based Ant Colony Routing Algorithm for Mobile Ad hoc Networks".

[8] Giovanni Comarela," Robot Routing in Sparse Wireless Sensor Networks with Continuous Ant Colony Optimization", GECCO'11, July 12-16, 2011, Dublin, Ireland. Copyright 2011 ACM 978-1-4503-0690-4/11/07

[39] C D'Souza," Implementation of Particle Swarm Optimization Based Methodology for Node Placement in Wireless Sensor Networks", International Conference and Workshop on Emerging Trends in Technology (ICWET 2011) - TCET, Mumbai, India ICWET'11, February 25-26, 2011, Mumbai, Maharashtra, India. ACM 978-1-4503-0449-8/11/02

[10] Taesoo Jun," Automated Routing Protocol Selection in Mobile Ad Hoc Networks", SAC'07 March 1115, 2007, Seoul, Korea ACM 1-59593-480-4/07/0003

[11] Jing-hui Zhong," Ant Colony Optimization Algorithm for Lifetime Maximization in Wireless Sensor Network with Mobile Sink", GECCO'12, July 7-11, 2012, Philadelphia, Pennsylvania, USA. ACM 978-1-4503$1177-9 / 12 / 07$

[12] Mohsen Saffarian," A Novel Protocol to Prevent Malicious Nodes from Misdirecting Forward Ants in AntNet Algorithm", SAC'08, March 16-20, 2008, Fortaleza, Ceará, Brazil. ACM 978-1-59593-753$7 / 08 / 0003$ 